Patrick Bouchet et Anne-Marie Lebrun, Management du tourisme sportif. De la consommation à la commercialisation

coll. "Des sociétés", Presses universitaires de Rennes, juin 2009

Jean Corneloup

\title{
OpenEdition
}

Journals

Édition électronique

URL : http://journals.openedition.org/tourisme/335

DOI : 10.4000/tourisme.335

ISSN : $2492-7503$

Éditeur

Éditions touristiques européennes

Édition imprimée

Date de publication : 1 juin 2010

Pagination : 86-87

ISSN : $2109-5671$

Référence électronique

Jean Corneloup, "Patrick Bouchet et Anne-Marie Lebrun, Management du tourisme sportif. De la consommation à la commercialisation », Mondes du Tourisme [En ligne], 1 | 2010, mis en ligne le 30 septembre 2015, consulté le 22 septembre 2020. URL : http://journals.openedition.org/tourisme/335 ; DOI : https://doi.org/10.4000/tourisme.335

Ce document a été généré automatiquement le 22 septembre 2020

Mondes du tourisme est mis à disposition selon les termes de la licence Creative Commons Attribution - Pas d'Utilisation Commerciale - Pas de Modification 4.0 International. 


\section{Patrick Bouchet et Anne-Marie Lebrun, Management du tourisme sportif. De la consommation à la commercialisation}

coll. “Des sociétés”, Presses universitaires de Rennes, juin 2009

Jean Corneloup

\section{RÉFÉRENCE}

Patrick Bouchet et Anne-Marie Lebrun, Management du tourisme sportif. De la consommation à la commercialisation, coll. "Des sociétés", Presses universitaires de Rennes, 2009.

1 Cet ouvrage sur le management du tourisme sportif fait suite à de nombreuses publications françaises dans ce domaine (Pigeassou, Sobry, Chazaud, Bessy, Bourdeau, Corneloup...). Sans aucun doute, la première impression porte sur l'importance des informations, références et ressources théoriques et pratiques sollicitées, tant françaises qu'anglo-saxonnes. Ces deux auteurs font preuve d'une grande maturité dans l'analyse du fait sportif touristique. Les ressources théoriques mobilisées touchent de nombreux domaines (gestion, sociologie, géographie, marketing, communication) et les références professionnelles, institutionnelles et territoriales sont variées. Cette maitrise du corpus, tant théorique que pratique, leur permet de présenter des concepts, des applications et des analyses et des exemples éclairant le propos, ainsi que des avancées théoriques en lien avec l'évocation de pistes de développement pratique. Enfin, il faut signaler leur volonté de circuler entre l'étude globale du tourisme et celle spécifique du tourisme sportif dans la perspective de montrer les continuités, les différences et les particularités de ce secteur. 
2 L'ouvrage s'articule en trois grandes parties qui servent de fil conducteur à leur "thèse" annonçant la nécessité de repenser la lecture des pratiques touristiques.

LA PREMIÈRE PARTIE commence par un historique sur le sport en montrant la polysémie du terme et la progressive montée de la sportivité des Français au cours des cinquante dernières années. Puis une description du secteur et des pratiques touristiques est proposée (économie, fréquentation, consommation...), ainsi que celle des mutations des consommations touristiques et de quelques pratiques émergentes (itinérance, tourisme de santé). Pour terminer, les auteurs qualifient le tourisme sportif en tant que secteur ayant ses propres caractéristiques et une place majeure aujourd'hui dans l'étude des pratiques touristiques. Contrairement à bien des études qui minimisent la place du sport, cet ouvrage souhaite montrer, à partir de différentes enquêtes, sa place montante et incontournable aujourd'hui. Cette approche structurelle, qui se "joue" des chiffres institutionnels (Odit, Credoc, Insep...), dresse un panorama comptable des fréquentations touristiques et des pratiques récréatives et sportives. Au-delà des querelles de chiffres, habituelles dans ce secteur et souvent liées à des imprécisions et à des variations dans la méthodologie des enquêtes, la richesse du contenu porte sur une description inhabituelle du tourisme sportif. Ce travail de précision terminologique sur la définition et la classification des pratiques de ce secteur, en référence aux écrits de nombreux chercheurs, est novateur. Les auteurs présentent aussi différentes familles de tourisme sportif (tourismes sportifs de spectacle, d'action, de culture et d'activisme) qui permettent de ne pas réduire ce secteur aux seules pratiques sportives de nature. De nouvelles pistes d'étude et d'analyse sont proposées, enrichissant considérablement le contenu des recherches et la place de ce secteur dans le tourisme global.

4 LA DeUXIÈme PARTIE concerne l'analyse "classique" de la demande et de l'offre de tourisme sportif. Le premier chapitre présente les modèles courants d'analyse des consommateurs en tourisme. La perspective consiste à utiliser dans un premier temps des données structurelles empruntées à la géographie (structure spatiale et espacetemps) et au marketing. Puis, dans un deuxième temps, d'autres modèles d'analyse du processus de consommation touristique sont abordés. Les références à la psychosociologie permettent d'évoquer les rôles de l'espace vécu, des interactions sociales et de la valorisation de soi dans l'analyse de ce processus. Une première ouverture théorique est ainsi proposée. Il s'agit de ne pas réduire l'analyse du processus de consommation touristique aux variables sociologiques et démographiques classiques (âge, sexe, lieu d'habitation, revenus...). Une approche plus affinée des mobiles de la pratique touristique est exposée. Elle souhaite montrer l'existence d'une spécificité esthétique attachée au tourisme sportif. Celui-ci serait plus disposé que les autres formes de pratique à la sensibilité individuelle et sociogéographique, présente dans l'activité vécue.

5 Les deux autres chapitres exposent des contenus sur la stratégie de l'offre entrepreneuriale et sur le marketing de la communication des entreprises et destinations touristiques. On quitte alors un monde pour deux autres. Il s'agit, dans un premier temps, d'exposer la variété de pratiques stratégiques en mouvement actuellement dans le tourisme (stratégies globales et typiques) et leur potentielle application en tourisme sportif en prenant comme exemple le tourisme des stations d'hiver. Dans un deuxième temps, l'objectif est de montrer l'importance, pour les acteurs touristiques, de travailler leur identité et leur marque sportive dans un souci de différenciation et de cohérence marketing dans le positionnement choisi. Ce chapitre 
sur la communication s'inspire de nombreux travaux provenant d'études publicitaires et en marketing stratégique pour souligner la variété des styles de communication possibles en fonction des finalités stratégiques envisagées. Les références marketing se distribuent entre l'attention portée aux attributs commerciaux du service (prix, qualité, avantages pratiques) et ceux mettant l'accent sur les aspects symboliques du service et du lieu de pratique. La spécificité du tourisme sportif porterait sur l'importance à accorder aux dimensions culturelles qui participent fortement à qualifier la valeur de l'offre attendue. Une subtile combinaison entre les différents attributs physiques et symboliques du produit, de la destination et du sport est préconisée. Pour terminer, la perspective théorique sur un plan stratégique exprimée par les auteurs consiste à mettre l'accent sur le dilemme entre la communication sur le produit ou la destination, le marché ou le territoire. La réponse proposée n'est pas tranchée. Elle dépend à la fois des styles touristiques des clients ou de visiteurs des lieux, mais aussi des liens possibles, historiques et actuels, entre le territoire et les opérateurs touristiques présents localement. Mais globalement, ce chapitre souhaite renforcer l'approche par le marketing de la communication en tourisme sportif en proposant différents apports théoriques et pratiques pour mieux analyser et comprendre ces activités professionnelles.

6 LA TROISIÈME PARTIE propose une ouverture théorique dans la façon de concevoir la pratique touristique. La prise en compte des expériences et des dimensions écologiques comme cadre de lecture du tourisme sportif doit s'imposer dans la manière de gérer les services proposés. La perspective théorique est stimulante, puisqu'elle envisage de repenser l'approche du consommateur en mettant en avant les aspects esthétiques, sensibles et expé- rientiels de la pratique. S'inspirant des théories post- modernes du social, le propos considère que l'individu est en attente de situations touristiques riches en participation, implication, exploration de soi et en enrichissement sensoriel. Dès lors, les prestations doivent se penser en fonction des expériences récréatives envisagées dans la déclinaison des produits, que ce soit au niveau de l'action sportive, de l'accueil, de l'ambiance, des relations sociales et spatiales ou de la mise en scène des supports promotionnels. De multiples exemples viennent étoffer cette lecture théorique en prenant comme objet le spectacle sportif, le tourisme d'action et les prestataires.

7 L'attention portée aux dimensions écologiques ouvre une autre perspective dans l'approche du tourisme sportif, en soulignant l'importance du détour par le territoire pour mieux organiser les liens entre les différentes parties prenantes. On passe ainsi d'une lecture expérientielle à une approche politique de ce secteur, en exposant les principes à développer pour améliorer les échanges dans la gestion des sites et la régulation écologique des lieux de pratique. Enfin, le dernier chapitre s'inscrit dans une approche projet en ingénierie touristique pour nous présenter la démarche conceptuelle, stratégique, opérationnelle et logistique à mettre en place lors de la création d'un produit innovant en tourisme sportif. À partir d'exemples concrets, les auteurs exposent l'imposante procédure à suivre pour élaborer et gérer une prestation de qualité.

8 Si la qualité de l'ouvrage n'est pas à remettre en cause, tant celui-ci est riche en références, analyses et en thèmes abordés, on peut tout de même se poser la question de la nécessité de vouloir inclure toutes ces approches dans une seule publication. C'est peut-être à ce niveau que se situe la limite à cet exercice, qui peut s'expliquer par le fait 
que le tourisme n'est pas une discipline scientifique. C'est au chercheur de construire son objet scientifique en fonction du projet de recherche et de publication envisagé. De même, à trop vouloir mixer, dans une même partie ou chapitre, le management, le marketing, l'entrepreneuriat, l'étude du tourisme du spectacle et du tourisme d'action, les auteurs proposent des combinaisons qui interpellent. N'était-il pas possible d'envisager un autre découpage qui aurait permis une approche théorique et thématique un peu moins éclatée ? Enfin, on peut s'interroger sur l'absence de liaison entre le chapitre sur les dimensions expérientielles de la pratique touristique et celui sur l'ingénierie touristique. Il aurait été logique d'aborder l'ingénierie par une entrée expérientielle pour innover dans la manière de concevoir l'ingénierie touristique de demain. 